\title{
Interactions between the transmembrane domains of CD39: identification of interacting residues by yeast selection
}

\author{
Sari Paavilainen, and Guido Guidotti* \\ Department of Molecular and Cellular Biology, Harvard University, 7 Divinity Avenue, Cambridge, MA 02138, USA \\ *Corresponding author's e-mail address: guidotti@fas.harvard.edu
}

Published online: October 13, 2014 (version 1)

Cite as: S. Paavilainen and G. Guidotti. ScienceOpen Research 2014 (DOI: 10.14293/S2199-1006.1.SORLIFE.AEEERM.v1)

Reviewing status: Please note that this article is under continuous review. For the current reviewing status and the latest referee's comments please click here or scan the QR code at the end of this article.

Primary discipline: Life sciences

Keywords: CD39, Transmembrane domains, Helical wheel, Hydrophobic moment, Packing moment

\begin{abstract}
Rat CD39, a membrane-bound ectonucleoside triphosphate diphosphohydrolase that hydrolyzes extracellular nucleoside tri- and diphosphates, is anchored to the membrane by two transmembrane domains at the two ends of the molecule. The transmembrane domains are important for enzymatic activity, as mutants lacking one or both of these domains have a fraction of the enzymatic activity of the wild-type CD39. We investigated the interactions between the transmembrane domains by using a strain of yeast that requires surface expression of CD39 for growth. Random mutagenesis of selected amino acid residues in the N-terminal transmembrane domain revealed that the presence of charged amino acids at these positions prevents expression of functional protein. Rescue of the growth of these mutants by complementary mutations on selected residues of the C-terminal transmembrane domain indicates that there is contact between particular faces of the transmembrane domains.
\end{abstract}

\section{INTRODUCTION}

Ectoapyrase activities are present in virtually all eukaryotic cells, starting with protozoa [1]. The ectoapyrases of vertebrates are called ectonucleoside triphosphate diphosphohydrolases (E-NTPDases). The active site of the E-NTPDases is extracytoplasmic, either on the cell surface or in the lumen of intracellular membrane compartments, and it catalyzes the hydrolysis of phosphoanhydride bonds of nucleoside tri- and diphosphates. The E-NTPDases are distinguishable from other membrane-bound ATPases by their broad substrate specificity, divalent cation $\left(\mathrm{Ca}^{2+}\right.$ or $\left.\mathrm{Mg}^{2+}\right)$ requirements, and insensitivity to P- and V- type ATPase inhibitors (for review see [2]). There are eight members of the E-NTPDase family, six with two transmembrane domains [3]. NTPDases 1, 2, 3, and 8 are located in the plasma membrane, while NTPDase 4 , also known as UDPase [4] and LALP70 [5], is in the Golgi membranes and lysosomal vacuoles; NTPDase 7 is present in intracellular membranes [6]. NTPDase 5 has been shown to be a secreted enzyme [7] and NTPDase 6 has been reported to be partially membrane-bound to Golgi membranes, presumably through the uncleaved signal sequence [8]; both have higher activity for nucleoside diphosphates than for nucleoside triphosphates.

CD39, NTPDase 1, originally identified on the surface of B lymphocytes, was the first vertebrate ectoapyrase identified and cloned $[9,10]$. Since CD39 is present principally on the surface of endothelial cells and it converts ATP directly into AMP without releasing ADP, its major function appears to be the inhibition of ADP-induced platelet aggregation when ATP is released in the circulatory system [11]. In fact, administration of a soluble form of CD39 reduces platelet aggregation [12].

CD39, a 511 amino acid polypeptide, is composed of two transmembrane domains near the $\mathrm{N}$ - and C-terminal ends, short cytoplasmic $\mathrm{N}$ - and C-terminal segments, and a large extracellular domain containing the active site $[9,13]$. The extracellular domain contains five regions conserved in all members of the ectoapyrase family: ACR1-5, where ACR is an abbreviation of Apyrase Conserved Region. ACR1 and ACR4 are similar to the $\beta$ - and $\gamma$-phosphate-binding domains of a family of cytoplasmic ATPases including actin, hsp70, and hexokinase [14]. The transmembrane domains affect the nucleotidase activity of the enzyme [15]. Mutants of CD39 lacking one or both transmembrane domains have between $10 \%$ and $20 \%$ of the activity of wild-type CD39. Furthermore, many detergents used to solubilize the protein, like Triton $\mathrm{X}$ $100, \mathrm{C}_{12} \mathrm{E}_{9}$, and octylglucoside, bring about a loss of $90 \%$ of the enzymatic activity, whereas digitonin does not alter the enzymatic activity. Importantly, CD39 is a monomer in the first group of detergents and a dimer in digitonin solutions; the soluble form of CD39 lacking both transmembrane domains is also a monomer. These results suggested that interactions between the transmembrane domains are required to form the active form of $\operatorname{CD} 39$ and that the dimer may be the active form of the enzyme. According to this 
hypothesis, there should be two types of transmembrane domain interactions, intrasubunit and intersubunit. While considerable evidence has supported the proposition that intact transmembrane domains in a lipid environment are required for native enzymatic activity and specificity $[15,16$, 17], the relationship of dimers to activity is less evident. CD39 mutants lacking either the $\mathrm{N}$ - or C-terminal transmembrane domain are still able to dimerize in digitonin solutions, but have only $20 \%$ of the native CD39 enzymatic activity. In solutions of dodecyl maltoside, cholate, and CHAPS, CD39 is principally a dimer and has $10-20 \%$ of the wild-type CD39 enzymatic activity.

Furthermore, the properties of the transmembrane domains of CD39 are unusual as they exhibit dynamic rotational motions linked to the enzymatic activity [18-20]. These motions are supported by the recent structural evidence obtained by the group of Norbert Strater [21].

These results led us to study the interactions between the transmembrane domains. The idea was that the examination of the amino acid sequence of these domains should indicate which surfaces of the transmembrane $\alpha$-helices face the lipid of the phospholipid bilayer and which face another transmembrane $\alpha$-helix. We tested the predictions by determining the effects on enzymatic activity of mutations of several amino acid residues in the transmembrane domains of CD39 expressed in a yeast strain that requires active CD39 for growth.

\section{EXPERIMENTAL PROCEDURES}

\section{Strains, media and reagents}

All DNA manipulations were performed using Escherichia coli strains DH5 $\alpha$ (supE44D lacU169 (80lacZDM15) hsdr17 recA1 endA1 gyrA96 thi-1 relA1) or GM 2198 (dam negative[22]). The Saccharomyces cerevisiae strain YMR4 (MAT $\alpha$ his3-11,15

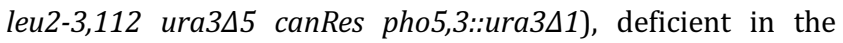
acid phosphatases PHO5 and PHO3 [23], was used for yeast manipulations.

Standard rich medium was used for E. coli [24]. Standard rich (YPD) and complete minimal uracil drop-out (DO-U) media were used for yeast [25]. The composition of the DO-U medium with $0.3 \mathrm{mM}$ ATP was $0.9 \mathrm{~g}$ of DO-U powder, $5 \mathrm{~g}$ of $\left(\mathrm{NH}_{4}\right)_{2} \mathrm{SO}_{4}, 1.02 \mathrm{~g}$ of $\mathrm{MgSO}_{4}-7 \mathrm{H}_{2} \mathrm{O}, 0.1675 \mathrm{~g}$ of $\mathrm{CaCl}_{2}, 0.1 \mathrm{~g}$ of $\mathrm{NaCl}, 0.55 \mathrm{~g}$ of $\mathrm{KCl}, 12.1 \mathrm{~g}$ of Tris base, and $0.165 \mathrm{~g}$ of ATP disodium salt (Sigma Aldrich) per liter of water; the $\mathrm{pH}$ was titrated to 7.2 with $\mathrm{HCl}$. Glucose (2\%), vitamins, and trace elements (DIFCO manual) were added after sterilization.

\section{Creation of an acid phosphatase-negative strain of S. cerevisiae}

Two dominant drug-resistance cassettes, kanMX4-module (pFA6a-kanMX4; [26]) and hphMX4-module (pAG32-hphMX4; [27]), were used to knock out completely the PHO11 and PHO12 genes, respectively, to create an acid phosphatasenegative (APN) YMR4 yeast strain. The selectable drug markers were polymerase chain reaction (PCR)-amplified using the following primers: PHO11fwd (5'-ATGTTGAAGTCA GCCGTTTATTCAATTTTACCGCTTCTTTGGTTAATGCACGTACGC TGCAGGTCGAC-3'), in which the first 51 nucleotides are the sense sequence of the PHO11 open reading frame (nucleotides 1-51) followed by the $5^{\prime}$-sense sequence of the kanMX4-module that is underlined; PHO11rev (5'-TAAAT CGGTATTCATATTTAGTTTCTAAAATTACATAATCATATTATCTA TCGATGAATTCGAGCTCG-3'), in which the first 50 nucleotides are the antisense sequence of the PHO11 gene just downstream of the stop codon (nucleotides 1406-1455) followed by the $5^{\prime}$-antisense sequence of the kanMX4 module that is underlined; PHO12fwd (5'-ATGTTGAAGTCAGCCGTTTATTC AATTTTAGCCGCTTCTTTGGTTAATGCCGGATCCCCGGGTTAATT AA-3') in which the first 50 nucleotides are the sense sequence of the $\mathrm{PHO} 2$ open reading frame (nucleotides 1-50) followed by the $5^{\prime}$-sense sequence of the hphMX4 module that is underlined; PHO12rev (5'-TTTGGACTGTGTTTGTTGTT CCAACTTGGTACTTCTCGCAAGCAAAAGTCCGATGAATTCXAGCT CGTTT- $3^{\prime}$ ) in which the first 50 nucleotides are the antisense sequence of the PHO12 gene 62 nucleotides downstream of the stop codon (nucleotides 1467-1516) followed by the 5 -antisense sequence of the hphMX4 module that is underlined. PCR products were transformed into $S$. cerevisiae YMR4, using the lithium acetate method [28]. Before plating transformants onto selective media, the cells were grown for $4 \mathrm{~h}$ in YPD at $30^{\circ} \mathrm{C}$, to allow for expression of the transformed drug resistance marker. Homologous integration of drug resistance cassettes was verified by PCR.

\section{Acid phosphatase assay}

Intact yeast cells were assayed for acid phosphatase activity as described by [29] with the following modifications. Yeast cells were grown in a liquid culture to an absorbance of 1 ; an aliquot of $100 \mu \mathrm{l}$ was centrifuged, the cells were washed once with $1 \mathrm{ml}$ of $0.1 \mathrm{M}$ acetate buffer $\mathrm{pH} 3.8$ and suspended in 60 $\mu \mathrm{l}$ of the acetate buffer. To this suspension, $60 \mu \mathrm{l}$ of $11 \mathrm{mM}$ $p$-nitrophenyl phosphate (Sigma Aldrich) in $0.1 \mathrm{M}$ acetate buffer $\mathrm{pH} 3.8$ was added and the reaction was allowed to proceed for $10-30 \mathrm{~min}$ at $30^{\circ} \mathrm{C}$. The reaction was stopped by removing the cells by centrifugation followed by the addition of $1.2 \mathrm{ml}$ of $0.2 \mathrm{M} \mathrm{NaOH}$. The absorbance was read at $401 \mathrm{~nm}$. One unit of acid phosphatase activity was defined as the amount of enzyme that catalyzes the liberation of $1 \mu \mathrm{mol}$ of p-nitrophenol per minute at $30^{\circ} \mathrm{C}$.

\section{Nucleotide phosphatase assay}

Nucleotide phosphatase activity was assayed as described by [30]. Yeast cells were washed twice with $50 \mathrm{mM}$ Tris- $\mathrm{HCl}, \mathrm{pH}$ 7.4, and suspended in the assay buffer containing $50 \mathrm{mM}$ Tris-HCl, pH 7.4, $1 \mathrm{mM} \mathrm{NaN}_{3}, 1 \mathrm{mM}$ EGTA, $1 \mathrm{mM} \mathrm{Na}_{3} \mathrm{VO}_{4}$, and $1 \mathrm{mM}$ ATP with or without $\mathrm{CaCl}_{2}$. The surface activity was assayed at room temperature for $30 \mathrm{~min}$. The $\mathrm{Ca}^{2+}$-stimulated 
ATPase activity was determined by measuring the inorganic phosphate released (31) and by subtracting values obtained without $\mathrm{CaCl}_{2}$ from those with $5 \mathrm{mM} \mathrm{CaCl}_{2}$.

\section{DNA constructions}

To express CD39 in yeast, a BamHI-digested fragment of pGZ131 [30] containing the entire CD39 gene was cloned into the pVT101-U vector [32]. Constructions of carboxy-terminal truncated (CT) and amino-terminal truncated (NT) CD39 plasmids are described before [15]. CT construct was digested with EcoRI and NotI and NT with HindIII and XhoI. 5' overhangs of the CT fragment were filled with Klenow fragment. CT and NT fragments were cloned into the pVT101-U vector.

To introduce single mutations into CD39, the entire gene of CD39 was cloned into a modified pYES2 [33]. The 5' overhangs of the BamHI fragment containing the entire CD39 was filled with Klenow fragment and cloned into the Xhol/HindIII site of pYES2 first filled with Klenow fragment.

Single random mutations were introduced at the amino acids G22, S24, and A34 of CD39 (the number refers to the number in the protein sequence) using the primers G22mut, S24mut, or A34mut, respectively, shown in Table 1 . The antisense primer (5'-TTCCATTCTGAGCAAGCG-3') contained an antisense sequence of CD39, nucleotides 402-420. The template was

Table 1. PCR primers used to create CD39 mutations. Primer sequence from $5^{\prime}$ to $3^{\prime}$

\begin{tabular}{|c|c|}
\hline G22mut & 5'-ATATTCTGATCATCCTTNNSTTCTCCTCTG -3' \\
\hline S24mut & 5'-ATATTCTGATCATCCTTGGTTTCNNSTCTGTCTTGG -3' \\
\hline 34mut & $\begin{array}{l}\text { 5'-ATATTCTGATCATCCTTGGTTTCTCCTCTGTCTTGGCTGTGAT } \\
\text { AGCTTTGATTNNSGTGGGACTGA - } 3^{\prime}\end{array}$ \\
\hline M2I & $\begin{array}{l}\text { 5'- GAAGGCTTCGAAAAGATGAACAGSNNTGTGATGACSNNG } \\
\text { GCGACSNNGACCAGGGAG -3' }\end{array}$ \\
\hline 1211 & $\begin{array}{l}\text { 5'- GAAGGCTTCGAAAAGATGAACAGCCCSNNGATGACCAT } \\
\text { SNNGACCAAGACSNNGGAGAAGAGA -3' }\end{array}$ \\
\hline 496 mut & 5'- GAAGGCTTCGAAAAGATGAACAGSNNTGTGATGACC -3' \\
\hline 495 mut & $\begin{array}{l}\text { 5'- GAAGGCTTCGAAAAGATGAACAGCCCSNNGATGAC } \\
\text { CATG }-3^{\prime}\end{array}$ \\
\hline M492mut & $\begin{array}{l}\text { 5'- GAAGGCTTCGAAAAGATGAACAGCCCTGTGATGACSNNG } \\
\text { GCGACCAAG - } 3^{\prime}\end{array}$ \\
\hline A491mut & $\begin{array}{l}\text { 5'-GAAGGCTTCGAAAAGATGAACAGCCCTGTGATGACCAT } \\
\text { SNNGACCAAGACC - } 3^{\prime}\end{array}$ \\
\hline 498Emut & 5'- GAAGGCTTCGAAAAGATCTCCAGCCCTGTG -3' \\
\hline 497 & 5'- GAAGGCT \\
\hline T495 & $\begin{array}{l}\text { 5'- GAAGGCTTCGAAAAGATGAACAGCCCCTCGATGAC } \\
\text { CATG -3' }\end{array}$ \\
\hline 1494Emut & $\begin{array}{l}\text { 5'- GAAGGCTTCGAAAAGATGAACAGCCCTGTCTCGACCAT } \\
\text { GGCG -3' }\end{array}$ \\
\hline V493Emut & $\begin{array}{l}\text { 5'- GAAGGCTTCGAAAAGATGAACAGCCCTGTGATCTCCATGG } \\
\text { CGACC - } 3^{\prime}\end{array}$ \\
\hline M492Emut & $\begin{array}{l}\text { 5' - AGGCTTCGAAAAGATGAACAGCCCTGTGATGACCTCGGCG } \\
\text { ACCAA - } 3^{\prime}\end{array}$ \\
\hline A491Emut & $\begin{array}{l}\text { 5'- GAAGGCTTCGAAAAGATGAACAGCCCTGTGATGACCATCT } \\
\text { CGACCAAGACC - } 3^{\prime}\end{array}$ \\
\hline
\end{tabular}

The BclI restriction sites are underlined and BstBI sites are double underlined. Randomly mutated nucleotides are shown in bold and underlined CTC codons show glutamic acid mutation.
pYE2S-CD39. Nucleotide combinations NNS in primers G22mut, S24mut, and A34mut, where N stands for any nucleotide and S for GMP and AMP, generate 32 different codons which code for all 20 amino acids and one stop codon. The PCR product was digested with BclI and SacII and the corresponding fragment in pYES2-CD39 was replaced with the random mutated G22, S24, or A34 PCR fragment. The resulting mutant libraries of G22mut, S24mut, or A34mut were digested with $S m a I$ and $\mathrm{XhoI}$ to cut out the whole mutated CD39 gene from pYES2-CD39 which was subcloned into the PvuII/XhoI site of pVT101-U. The resulting plasmids of G22mut, S24mut, or A34mut were transformed into APN $S$. cerevisiae YMR4. Transformants were plated onto DO-U plates and were grown for three days at $30^{\circ} \mathrm{C}$. Colonies were replicaplated onto $0.3 \mathrm{mM}$ ATP-DO-U plates. After three days, the growing colonies were streaked onto $0.3 \mathrm{mM}$ ATP-DO-U plates to verify the growth of mutants. Mutants were screened by yeast colony PCR (see later in this part). Selected mutant plasmids were isolated from yeast colonies, amplified in $E$. coli DH5 $\alpha$ and retransformed into yeast to confirm the growth.

To generate the library of rescue mutants of CD39, the entire CD39 gene was cloned into the BamHI/HindIII site of pBluescript II vector. A primer CD39BstBI-mut, containing a sense sequence of CD39 (nucleotides 1466-1513), an NcoI and a BstBI restriction site (5'-TGGTCGCCATGGTCATCAC AGGGCTGTTCATCTTTtcgAAGCCTTCGT-3', the restriction sites are underlined, at the BstBI site the lowercase letters represent the changes from the original bases), and pVT101rev primer (5'-GCAAGGTAGACAAGC-3'), containing an antisense sequence of pVT101-U [32], was used in PCR to introduce a BstBI restriction site into CD39. The template was pVT101-CD39. The resulting PCR product was digested with NcoI and BamHI, and the corresponding fragment in pBluescript-CD39 was replaced with this NcoI/BamHI PCR fragment.

The libraries of G22E or G22R rescue mutants were generated with pVT101fwd primer (5'-CTGCACAATATTTCAAGC-3') and an antisense primer M2I containing a BstBI site and an antisense sequence encoding CD39 complementary to nucleotides 1455-1511 in which L489, M492, and G496 were randomly mutated (Table 1). The templates were pVT101CD39G22E or G22R. Rescue PCR products of S24E or S24R mutants were generated with pVT101fwd primer and an antisense primer M2II containing a BstBI site and an antisense sequence of CD39 complementary to nucleotides 1449-1511 in which L487, A491, and T495 were randomly mutated (Table 1). The templates were pVT101-CD39S24E or S24R. PCR products were cloned into the BclI/BstBI site of pBluescript-CD39. The libraries of $\mathrm{G} 22 \mathrm{E}$ and $\mathrm{G} 22 \mathrm{R}$ rescue mutants were subcloned into the BamHI/HindIII site of pVT101-U and rescue mutants were selected on $0.3 \mathrm{mM}$ ATPDO-U plates as explained earlier in the experimental part.

The rescue libraries of G22E or G22R with a single amino acid were generated with pVT101fwd primer and an 
antisense primer G496mut or M492mut (Table 1) with pVT101-CD39G22E or R as a template. Single rescue mutants of S24E or R were generated with pVT101fwd primer and an antisense primer T495mut or A491mut (Table 1) and with pVT101-CD39S24E or R as a template. PCR products were cloned into the BclI/BstBI site of pBluescript, subcloned into the BamHI/HindIII site of pVT101-U, and selected on $0.3 \mathrm{mM}$ ATP plates as explained earlier. The specificity of G22R or S24R rescue was tested with constructs which were created using pVTfwd primer and an antisense primer F498E, L497E, T495E, I494E, V493E, M492E, or A491E (Table 1) and pVT101-CD39G22R or S24R mutant plasmids as templates. PCR products were cloned into the Bcll/BstBI site of pBluescript and subcloned into the BamHI/HindIII site of pVT101-U.

To introduce an HA tag into the C-terminal end of wtCD39, G22R, or G22R,M492T,G496E, a primer CD39-tag, containing an antisense sequence of CD39 (nucleotides 1515-1533), BamHI restriction site, and HA tag (5'-GGTACCGGATCCTC ACAGAGAAGCGTAGTCTGGGACGTCGTATGGGTATACTGCCTCT TTCCAGAAA-3', BamHI underlined), and pVT101fwd primer were used with pVT101-CD39, G22R or G22R,M492T,G496E as templates to amplify the entire gene. PCR products were cloned into the BamHI/HindIII site of pVT101-U.

\section{Yeast colony PCR}

Yeast colonies were picked up with pipet tips and mixed into $45 \mu \mathrm{l}$ of $0.25 \%$ SDS. A total of $50 \mu \mathrm{l}$ of acid-washed glass beads were added into tubes and samples were vortexed for $4 \mathrm{~min}$. After vortexing, the samples were centrifuged for 3 min. Supernatants were diluted 25 -fold with water and $2 \mu \mathrm{l}$ of the dilution was used for PCR.

\section{Yeast crude membrane preparation}

Cells were grown to $\mathrm{A}_{600} \sim 2.0$ in DO-U medium containing $2 \%$ glucose at $30^{\circ} \mathrm{C}$. Yeast crude membranes were isolated as described previously [34].

\section{Immunoblots}

Western blot analysis was done as described before [18]. Samples were resolved on a 7.5\% SDS-polyacrylamide gel. Nitrocellulose membranes were probed with anti-HA11 monoclonal antibody (Covance). Protein concentration was determined with the protein assay kit of Bio-Rad Laboratories (Hercules).

\section{RESULTS \\ Expression of wtCD39, $\triangle \mathrm{TM} 1 \mathrm{CD} 39$, and $\triangle \mathrm{TM} 2 \mathrm{CD} 39$ mutants in yeast}

In order to use yeast growth as a measure of the expression of active CD39, we constructed a strain of $S$. cerevisiae lacking all four acid phosphatase genes and used a medium with ATP as the only source of phosphate. Since nucleotides are unable to enter the cell [35], the yeast strain would be able to grow only if enzymatically active CD39 is present on the surface of the cells. The acid phosphatase activity at $\mathrm{pH} 3.8$ of the parent YMR4 strain was $0.14 \pm 0.006$ unit $/ 10^{8}$ cells, whereas the activity of the deletion strain was $0.001 \pm 0.001$ unit $/ 10^{8}$ cells indicating the absence of phosphatase activity on the intact cells.

Growth of the APN S. cerevisiae strain on a solid DO-U medium containing ATP as a phosphate source is shown in Figure 1. It is evident that yeast transformed with a plasmid containing wtCD39 grew more rapidly than the yeast containing the control vector. This result supported the view that

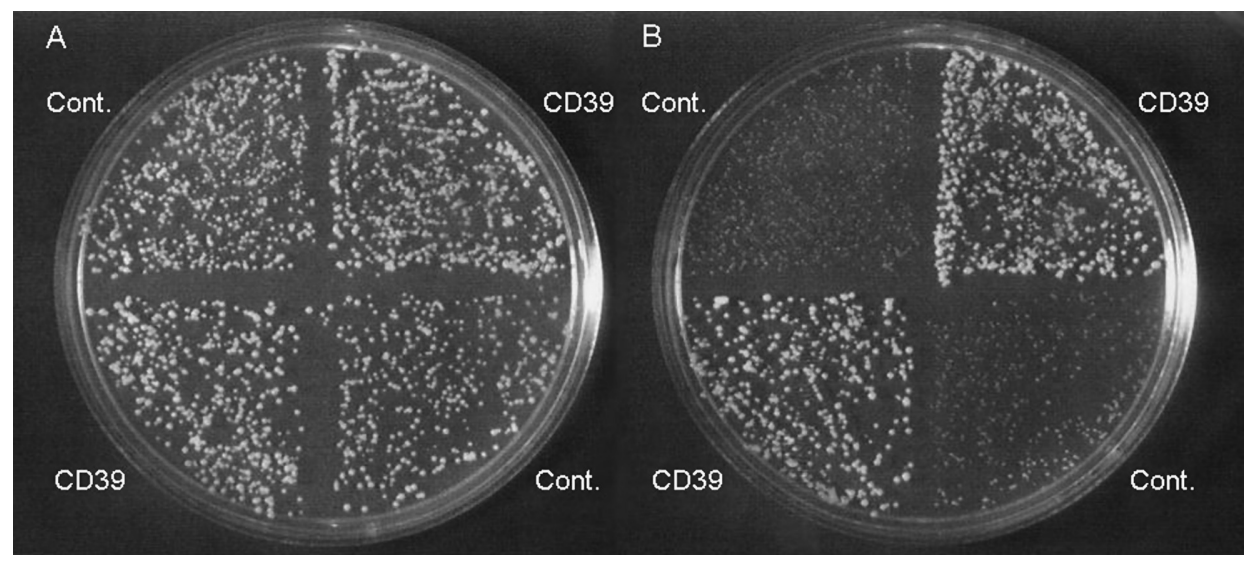

Figure 1. Growth of acid phosphatase-negative yeast strain on uracil-deficient medium supplemented with phosphate or ATP. The cells of the yeast strain carrying pVT101-CD39 (CD39) or pVT101 (Cont.) were streaked on a synthetic minimal DO-U medium plate containing phosphate (A) or $0.3 \mathrm{mM} \mathrm{ATP}$ at $\mathrm{pH} 7.2$ as phosphate source (B). A total of $10 \mu \mathrm{l}$ of a cell culture $\mathrm{OD}_{650}=0.1$ was plated on both plates. The plates were incubated at $30^{\circ} \mathrm{C}$ for 3 days. 


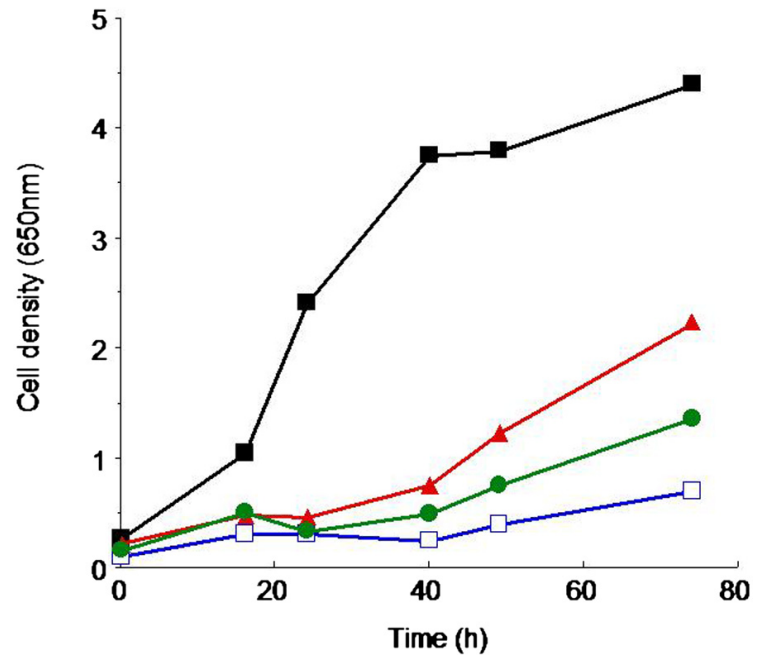

Figure 2. Growth of acid phosphatase-negative yeast strain in liquid uracil-deficient medium containing ATP. Yeast carrying

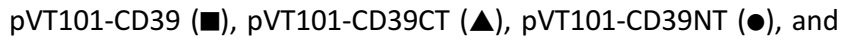
pVT101 ( $\square)$ were grown in DO-U medium, $\mathrm{pH} 7.2$ at $30^{\circ} \mathrm{C}$ containing $0.3 \mathrm{mM}$ ATP.

wtCD39 was expressed at the surface of yeast cells and thus was able to hydrolyze ATP. Intact control cells had no measurable ATPase activity at $\mathrm{pH} 7.4,0.16 \pm 0.17$ nmole $/ \mathrm{m} / 10^{8}$ cells, while the ATPase activity of cells expressing wtCD39 was $2.55 \pm 0.63 \mathrm{nmole} / \mathrm{m} / 10^{8}$ cells. The slow growth of the control strain was caused by the slow spontaneous hydrolysis of ATP.
The growth of wtCD39 and the mutants lacking the $\mathrm{N}$ - or C-terminal transmembrane domains ( $\triangle \mathrm{TM} 1 \mathrm{CD} 39$ and $\triangle$ TM2CD39) [15] was measured in a liquid ATP-DO-U medium at $\mathrm{pH}$ 7.2. The medium was buffered to slow down the decrease of $\mathrm{pH}$ caused by acid production during fermentation so that the enzymatic activity of CD39 could operate near its $\mathrm{pH}$ optimum [36]. The growth curves shown in Figure 2 indicate that wtCD39 grew faster than the $\triangle \mathrm{TM} 1 \mathrm{CD} 39$ and $\triangle \mathrm{TM} 2 \mathrm{CD} 39$ mutants. The growth rates roughly followed the enzyme activity of CD39 and its mutants. The activity of the $\triangle \mathrm{TM} 1 \mathrm{CD} 39$ and $\triangle \mathrm{TM} 2 \mathrm{CD} 39$ mutants was about $35 \%$ and $25 \%$, respectively, of the activity of the wtCD39 when measured three days after inoculation. These results indicated that the yeast strain expressing CD39 could be used to assess the enzymatic activity of CD39 at the surface of the cell.

\section{Selection of functional TM1 and TM2 CD39 mutants}

The aim of this research was to determine whether there are protein-protein interactions between TM1 and TM2 of CD39 in order to understand how the transmembrane domains affect the enzymatic activity of the nucleotidase.

We determined the helical hydrophobic moment of the transmembrane helices according to [37] and identified the faces of the helices that are most hydrophobic and presumably are exposed to lipid. These are shown in Figure 3 as a black vector on the helical wheel diagrams. We then calculated the helix packing moments for the transmembrane domains according to [38], in order to identify the faces of TM1 and

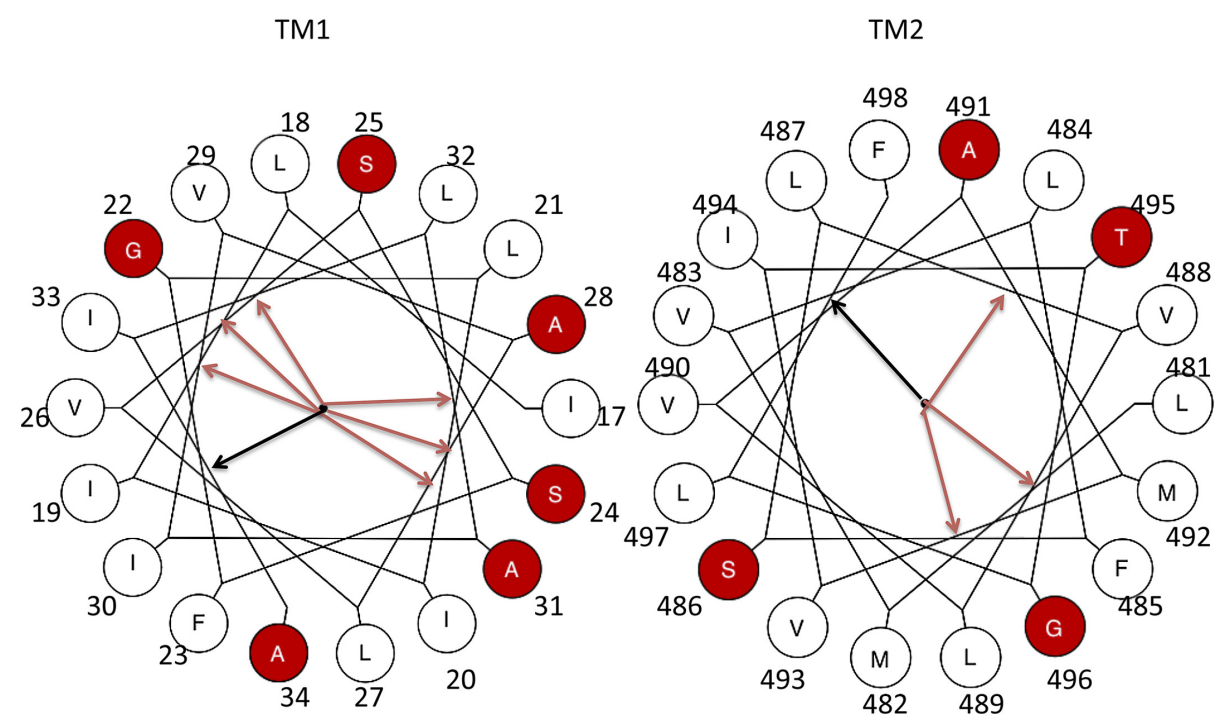

Figure 3. Helical wheel projections of TM1 and TM2 of CD39 using the program of Kael Fisher (http://kael.net/helical.htm). TM1, starting at $\mathrm{A} 34$, and TM2, starting at L481, are shown going from the extracellular surface to the cytoplasmic surface of the plasma membrane. The residues colored red have helix packing values larger than that of $\mathrm{Gln}$ (38). The black arrow indicates the direction of the hydrophobic moment (37); the red arrows show residues with helix packing moments greater than that of Gln suggesting helical packing interfaces (38). 
TM2 that are likely to participate in helix interfaces. These regions appear to be rich in small and weakly polar residues [39]. The helix packing moments, shown in Figure 3 as red vectors, indicates that two faces of TM1 and TM2 could participate in contacts between helices. To investigate the possibilities presented by the results in Figure 3, we randomly mutated three residues of TM1, G22, and S24 at two potential interhelical contact faces, and A34 on a lipid exposed face, to see which substitutions were compatible with expression of active CD39. Each mutant library was transformed into the APN S. cerevisiae. About 50-60 growing colonies of each mutant library (G22, S24, or A34) were sequenced to verify which amino acid allowed a functional CD39. Also eight to nine slowly growing yeast colonies of each mutant type were sequenced to determine amino acid substitutions that do not allow expression of active CD39. Table 2 shows the results of these random mutations.

Three types of amino acid substitutions were found for all three sites. The largest group comprises the substitutions that allow production of functional CD39 and includes $G$, the nonpolar amino acids (A, L, V, I, and M), S, T, C, F, and W. A second group includes $\mathrm{P}, \mathrm{K}, \mathrm{R}, \mathrm{H}, \mathrm{D}, \mathrm{E}, \mathrm{N}$, and $\mathrm{Q}$ that were not permissive for production of functional CD39. The reason that no colonies, even slow growing, with these mutations were isolated at some sites, for instance, $\mathrm{K}$ and $\mathrm{H}$ at all three sites, may be the consequence of insufficient sampling of slow-growing colonies (only 25). The third group is $\mathrm{Y}$ that allows growth at A34 but prevents production of active CD39 at G22 and S24.
Since the hypothesis was that G22 and S24 are likely to be at helix contact sites, it was surprising that their replacement with large amino acids like L, V, I, M, F, and W had no effect on expression of active CD39; whereas the substitution of these residues at $\mathrm{A} 34$ was compatible with the view that $\mathrm{A} 34$ faces the lipid environment. Interestingly, the G22Y and S24Y mutants grew more slowly on an ATP plate than wtCD39 and the A34Y mutant. Tyrosine differs from other aromatic amino acids in that it has a polar hydroxyl group, which may interfere with helix packing; in any event its occurrence at helix interfaces is low [39].

All Pro-mutants of CD39 were inactive, a situation compatible with the known disruption of the $\alpha$-helix by the ring structure of Pro. Although it was not unexpected that substitution of G22, S24, and A34 with the charged amino acids K, R, H, D, and E prevented expression of active CD39, it was not clear why the polar $\mathrm{N}$ and $\mathrm{Q}$ were also deleterious for growth.

Table 2 also shows the expected and calculated fractional appearances of the different amino acids. Ala, Ser, and Arg have the highest expected fractional appearance because each of them are represented by 3 of the 32 codons in the random mixture. Gly, Leu, Val, Pro, and Thr are represented by 2 of the 32 codons and the other amino acids have one codon in the random mixture.

Since selection was based on the rate of growth of the colonies on plates, it is possible that the explanation for the higher frequency of appearance of some amino acids compared to others, for instance, S24G compared to S24L, is a consequence of different growth rates of these colonies. However, since the

Table 2. Amino acid substitutions at three amino acid positions in TM1 of CD39

\begin{tabular}{|c|c|c|c|c|c|c|}
\hline \multirow[b]{2}{*}{ Amino acid } & \multicolumn{3}{|c|}{ Allowed changes } & \multicolumn{3}{|c|}{ Disallowed changes } \\
\hline & G22 & S24 & A34 & G22 & S24 & A34 \\
\hline $\mathrm{G}(0.062)$ & $16(0.25)$ & $11(0.172)$ & $5(0.078)$ & & & \\
\hline A (0.094) & $3(0.047)$ & $5(0.109)$ & $2(0.032)$ & & & \\
\hline L (0.062) & $10(0.156)$ & $4(0.065)$ & $13(0.204)$ & & & \\
\hline$V(0.062)$ & $13(0.204)$ & $7(0.14)$ & $5(0.078)$ & & & \\
\hline I (0.031) & & $3(0.047)$ & $6(0.094)$ & & & \\
\hline $\mathrm{M}(0.031)$ & $1(0.016)$ & $2(0.032)$ & $4(0.062)$ & & & \\
\hline$P(0.062)$ & & & & $1(0.016)$ & $1(0.016)$ & $1(0.016)$ \\
\hline$S(0.094)$ & $5(0.078)$ & $5(0.078)$ & $5(0.078)$ & & & \\
\hline $\mathrm{T}(0.062)$ & $2(0.032)$ & $4(0.0625)$ & $9(0.14)$ & & & \\
\hline$C(0.031)$ & $1(0.016)$ & $6(0.094)$ & & & & \\
\hline$F(0.031)$ & $2(0.032)$ & $5(0.047)$ & $6(0.094)$ & & & \\
\hline W (0.031) & $3(0.047)$ & $4(0.0625)$ & $3(0.047)$ & & & \\
\hline$Y(0.031)$ & & & $6(0.094)$ & $1(0.016)$ & & \\
\hline \multicolumn{7}{|l|}{$\mathrm{K}(0.031)$} \\
\hline$R(0.094)$ & & & & $2(0.032)$ & & $1(0.016)$ \\
\hline \multicolumn{7}{|l|}{$H(0.031)$} \\
\hline $\mathrm{D}(0.031)$ & & & & & $1(0.016)$ & $2(0.032)$ \\
\hline$E(0.031)$ & & & & $4(0.062)$ & $3(0.047)$ & $3(0.047)$ \\
\hline $\mathrm{N}(0.031)$ & & & & & $1(0.016)$ & $1(0.016)$ \\
\hline$Q(0.031)$ & & & & & $2(0.032)$ & $1(0.016)$ \\
\hline Altogether & & & & 64 & 64 & 73 \\
\hline
\end{tabular}

The first column indicates amino acids and their expected fractional appearances in parentheses. Next three columns show amino acids changes which gave active CD39 and their calculated fractional appearances based on the total number of amino acids at the mutated position. Last three columns show amino acids which were detrimental for CD39 function 
sample size for each site was only twice the number of possible codons, the measured frequency of appearance of a given amino acid at each site is not an accurate measure of the actual frequency that would be obtained with a much larger sample size.

Random mutagenesis was also done at position A491 of TM2. Mutants with substitutions of charged amino acids, Pro and all the aromatic amino acids at this position, did not allow the production of active CD39. Clearly, some helical positions are more sensitive to the quality of the side chain.

We also investigated the effect of changes from a large side chain to a small one on the properties of CD39. Mutations of I33 and F485 to $G$ decreased the expression of functional CD39, indicating that these big amino acids cannot be mutated to a small one without loss of activity.

We conclude that the regions of TM1 with large packing moments can tolerate replacement with most other amino acids except for those with charge.

\section{Selection of TM2 mutants that rescue TM1 mutants that are not viable}

As indicated in Table 2, the substitution of charged amino acids at G22 and S24 of CD39 does not allow the growth of transformed yeast. Accordingly, we constructed four plasmids with CD39 mutations (G22E, G22R, S24E, and S24R) in TM1 and verified that they do not confer viability on transformed yeast strains. To investigate whether these mutated residues interact with residues of TM2, we made random mutations of residues of TM2 which on the basis of the information in Figure 3 might interact with G22 and S24, to wit L489, M492, and G496 of TM2 for G22 and L487, A491, and T495 of TM2 for S24. The helix net diagrams [40] shown in Figure 4 indicate the rationale for the pairings. Table 3 shows the results of these experiments; also included in the Table are the surface ATPase activities of the yeast cells transformed with the various mutated plasmids.

G22E was rescued by three types of mutants: G496 was a positively charged amino acid and M492 was Ser or Leu; G496 and/or M492 were/was Ser; and L489 was Pro while G496 was Leu, Tyr, or Trp and M492 was A or L; the latter result suggests that the effect of the Pro mutation on the structure of the $\alpha$-helix is important. G22R was rescued by a positively charged residue at G496 and a polar residue at M492, or by a Pro at L489 with a polar residue at either 492 or 496. The striking feature of the results is that all the mutations that restored the functional expression of the G22E and G22R CD39 mutants had a polar or a charged residue at M492 or G496 or at both positions. The only exception was the rescue of G22E by L489P, M492A, and G496L. With the
TM2

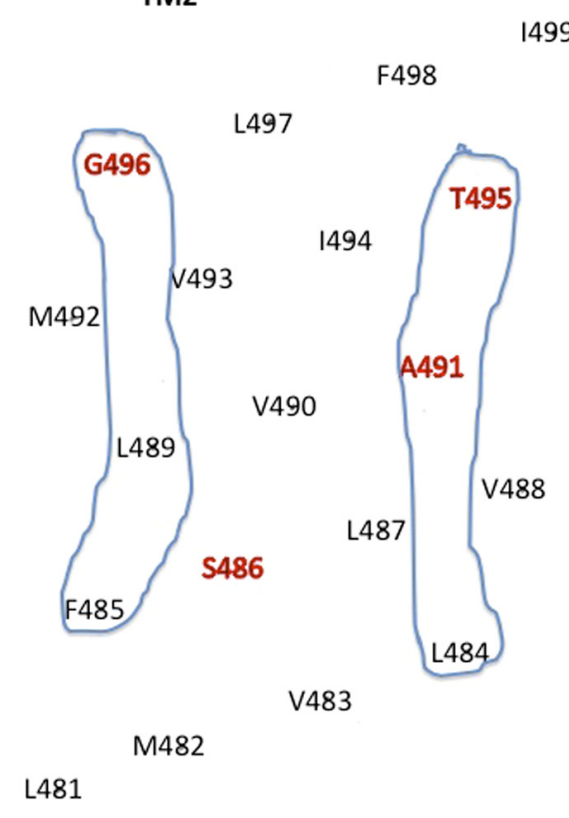

1499

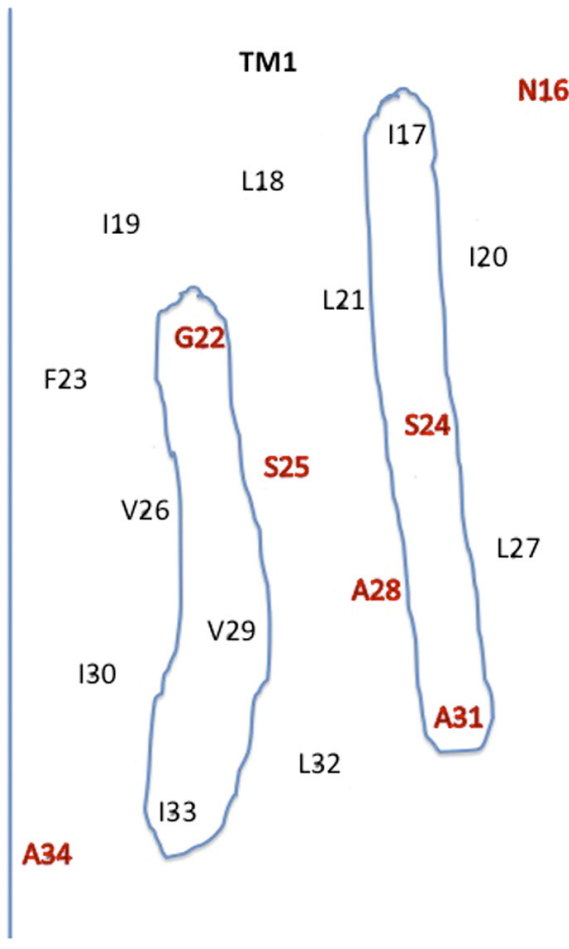

Figure 4. Helical net diagrams (40) of TM1 and TM2. The transmembrane domains are shown with the extracellular surface at the bottom and the cytoplasmic surface at the top of the figure. The residues colored red have helix packing values larger than that of Gln (38). The residues grouped by blue lines in TM1 and TM2 correspond to those in Figure 3 indicated by the red arrows suggesting helical packing interfaces. 
Table 3. The rescue of glutamic acid or arginine mutants in the TM1 with the mutations in the TM2 of CD39

\begin{tabular}{|c|c|c|c|c|c|c|c|c|c|}
\hline \multirow[b]{2}{*}{ G22E } & \multicolumn{2}{|c|}{$\begin{array}{c}\text { Amino } \\
\text { acids in TM2 }\end{array}$} & \multicolumn{2}{|c|}{$\begin{array}{c}\text { Enzyme } \\
\text { activity (\% of WT) }\end{array}$} & \multirow[b]{2}{*}{ S24E } & \multicolumn{2}{|c|}{ Amino acids in TM2 } & \multicolumn{2}{|c|}{ Enzyme activity (\% of WT) } \\
\hline & L489 & M492 & G496 & $25-30$ & & L487 & A491 & T495 & $15-20$ \\
\hline \multirow[t]{9}{*}{ G22E rescue } & L & $S$ & $\mathrm{H}$ & & S24E rescue & M & $\mathrm{T}$ & $\mathrm{T}$ & \\
\hline & A & $S$ & H & $35-40$ & & L & $\mathrm{T}$ & $\mathrm{S}$ & \\
\hline & A & L & H & & & 1 & $\mathrm{~T}$ & v & $40-50$ \\
\hline & L & L & $\mathrm{R}$ & & & $\mathrm{L}$ & $\mathrm{S}$ & $\mathrm{S}$ & \\
\hline & L & $\mathrm{s}$ & $\mathrm{L}$ & $70-80$ & & & & & \\
\hline & L & $\mathrm{S}$ & $S$ & & & & & & \\
\hline & $\mathrm{P}$ & A & $\mathrm{L}$ & & & & & & \\
\hline & $P$ & L & Y & $45-50$ & & & & & \\
\hline & $P$ & $\mathrm{~L}$ & W & & & & & & \\
\hline G22R & L489 & M492 & G496 & $15-20$ & S24R & L487 & A491 & T495 & $10-15$ \\
\hline \multirow[t]{4}{*}{ G22R rescue } & L & $\mathrm{T}$ & $\mathrm{E}$ & $25-40$ & S24R rescue & \multirow{2}{*}{\multicolumn{4}{|c|}{ No rescue mutants }} \\
\hline & G & C & D & & & & & & \\
\hline & $P$ & L & $\mathrm{T}$ & & & & & & \\
\hline & $\mathrm{P}$ & Y & A & & & & & & \\
\hline
\end{tabular}

exception of the latter case, a possible explanation of these results is that the residues substituted at M492 and G496 are capable of interacting with G22E by hydrogen bonding or salt linkages. Interestingly, the M492S mutation was more effective than the $\mathrm{G} 496 \mathrm{H}$ or $\mathrm{R}$ mutations in its rescue ability and the ATPase activity of the Ser rescue mutants was about twice the activity of $\mathrm{G} 496 \mathrm{H}$ or $\mathrm{R}$ rescue mutants (Table 3). The G22R mutation was rescued by the presence of Asp or Glu at G496 and of Thr or Cys at M492, and by the presence of Pro at L489 and Thr or Tyr at G496 or M492 (Table 3).

S24E was rescued with Thr or Ser at A491 and/or at T496. The various combinations suggest that a polar residue at A491 or T496 is necessary, since the A492T, T496V mutant is viable. We conclude that in wtCD39, S24 interacts with T496. The activity of the S24E mutant was about $15 \%$ of that of wtCD39, while the rescue mutants had about $40-50 \%$ of wtCD39 activity. No rescue mutations involving the presence of charged amino acids were found. No mutations were found that could rescue the S24R mutant.

In order to determine whether the mutations at M492, G496 and A491, T495 individually are able to rescue the G22E, G22R and the S24E, S24R mutations, respectively, random mutagenesis of M492 and G496 and of A491 andT495 was done and colonies were selected for growth on ATP plates. The results are shown in Table 4. The activity of the G22E mutant was restored by substitution of Ser or Cys at M492 and by substitution of G496 by Ser, verifying the conclusion that a polar residue has to be present at one of these sites. Interestingly, no single positively charged amino acid substitution of G496 could restore the activity of the G22E mutant, although the results in Table 3 show that the combinations L489A/M492L/G496H and L489L/M492L/G496R were able to rescue the G22E mutant. On the other hand, the $G 22 \mathrm{R}$
Table 4. The rescue of glutamic acid or arginine mutants in TM1 by single amino acid mutations of TM2

\begin{tabular}{cccccc}
\hline \multicolumn{5}{c}{ Amino acid } \\
\hline & M492 & G496 & & A491 & T495 \\
\hline G22E rescue & S or C & S & S24E rescue & Tor S & no mutants \\
G22R rescue & Y or T & E & S24R rescue & no mutants & no mutants \\
\hline
\end{tabular}

mutant was rescued by G496E, as well as by either Y or T at M492. The S24E mutant was rescued by A491T or S, a result supporting the view that a polar residue at this site is required for rescue; no single mutants of T495 were found. No rescue mutations at A491 and T495 were found for the S24R mutant.

We conclude that the face of the TM1 helix containing G22 interacts with the face of TM2 containing M492 and G496, while the face of TM1 with S24 interacts with the face of TM2 with A491 and T496.

\section{Specificity of the interactions between G22 and G496}

The hypothesis that the TM1 and TM2 transmembrane helices interact at the faces containing G22 and G496 was explored by asking whether the G22R mutation was rescued specifically by substitution of G496 by Glu rather than by replacement of any residue between A491 and F498 by Glu. Only the G496E mutation was able to restore the activity of the G22R mutant confirming that the interaction between G22R and G496E is specific. None of these single-site mutations were able to rescue the S24R mutant supporting the specificity of the preceding result. 


\section{Expression of wtCD39 and CD39 mutants in yeast}

The expression of wtCD39, the G22R mutant, and the G22RM492T, G496E rescue mutant in yeast cells was examined by the separation of crude yeast membranes by SDS-PAGE and immunoblotting with anti-HA antibody (Figure 5). Samples were also deglycosylated with glycopeptidase $F$ to ensure that glycosylation had taken place.

Cell membranes from yeast transformed with plasmids containing CD39 cDNA had a band in the range between 56 and $64 \mathrm{kDa}$. The membranes with wtCD39 had the principal band at $64 \mathrm{kDa}$ (lane 5). Most of the CD39 protein in the membranes from the G22R mutant, that is deficient in ATPase activity, was in the $56 \mathrm{kDa}$ region; however, a smear of protein approached close to the $64 \mathrm{kDa}$ region (lane 1). However, the membranes of yeast expressing the G22R-M492T, G496E rescue mutant with twice the ATPase activity of the G22R mutant had a well-defined band at $64 \mathrm{kDa}$ (lane 3). None of these bands were present in the yeast cells transformed with the empty vector (lane7) supporting the view that the bands are CD39 protein.

After deglycosylation of the membrane protein with glycopeptidase $\mathrm{F}$, all three samples had bands around $56 \mathrm{kDa}$, which corresponds roughly to the correct molecular mass of the unglycosylated wtCD39 [41]. No $56 \mathrm{kDa}$ protein band was detected in the control. Clearly, all three proteins were glycosylated; while wtCD39 was extensively processed, the mutant proteins were in large part not glycosylated and retained in the endoplasmic reticulum. Additional bands from 18 to 36 $\mathrm{kDa}$ were recognized in the samples of CD39 and the mutants

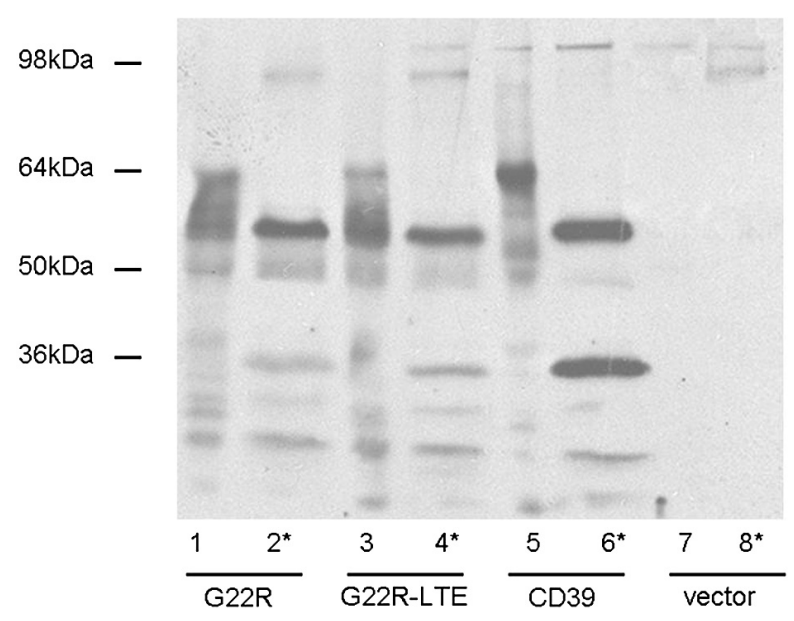

Figure 5. Expression of wtCD39, and the G22R and G22R-LTE (L489L, M492T, G496E) mutants in yeast. A total of $15 \mu \mathrm{g}$ of crude membrane proteins of samples pVT101-CD39G22R (lanes 1-2), pVT101-CD39 G22R-LTE (lines 3-4), pVT101-CD39 (lanes 5-6), and pVT101 (lanes 7-8) were treated $(*)$ or not treated with glycopeptidase $\mathrm{F}$. The samples were subjected to $7.5 \%$ SDS-PAGE and examined by immunoblotting with anti-HA11 monoclonal antibody. but not in the control sample. These bands are degradation products which were formed during the isolation of cell membranes.

\section{DISCUSSION}

We have constructed the yeast strain APN that requires the presence of active CD39 at the cell surface to grow on media with ATP as the only source of phosphate. The strain was derived from YMR4, deficient in the PHO3 and PHO5 acid phosphatase genes, by deletion of the PHO11 and PHO12 acid phosphatase genes. The surface ATPase activity of APN was $0.16 \pm 0.17$ nmole $/ \mathrm{m} / 10^{8}$ cells, whereas APN transformed with a plasmid containing the cDNA for wtCD39 had a surface ATPase activity of $2.55 \pm 0.63 \mathrm{nmole} / \mathrm{m} / 10^{8}$ cells, comparable to that of YMR4. In liquid media, the growth rate of APN was negligible in the absence of transformation with the wtCD39 plasmid (Fig. 2). On agar plates, there was sufficient spontaneous hydrolysis of ATP that APN grew slowly but at an easily distinguishable rate from that of the cells transformed with the wtCD39 plasmid.

We used this yeast strain to investigate the effects of mutations of residues in the transmembrane helical domains on the surface expression of enzymatically active CD39. By random mutagenesis, a library of mutants of a particular amino acid was made and selected for expression of CD39 adequate for growth on ATP plates.

The central question was whether interactions between the transmembrane helices of CD39 could be identified. The approach was based on the view that the surfaces of TM1 and TM2 defined by the hydrophobic moment would be likely to be exposed to lipid [37], while the helix surfaces characterized by helix packing moments would be involved in interactions between the transmembrane helical domains [39].

The three small amino acid residues of TM1 selected for mutational analysis are located in the second, third and fifth turns of the $\alpha$-helix from the cytoplasmic border. The result that all amino acids with a positive value on the hydrophobicity scale of [42] can substitute for A34 is compatible with the possibility that this residue is at a non-interfacial position, as is also suggested by the hydrophobic moment and by the ability of Tyr, a residue with a high propensity for noninterfacial localization, to substitute for A34. On the other hand, the result that residues with a large hydrophobic side chain can substitute for G22 and Ser24 appears to compromise the view that these amino acids are on interfacial faces. The inability of Tyr to replace G22 and S24, however, supports this view. We conclude that G22 and S24 are likely to be on interfacial faces of TM1 and that the $\alpha$-helices in question are likely to be closer to one another at the extracellular ends and farther apart at the cytoplasmic ends to account for the ability of large nonpolar amino acids to occupy these positions in a functional protein.

Previous results from this laboratory have suggested that the transmembrane helices are highly mobile and that this mobility is a prerequisite for function [18-20]. We investigated this 
premise by rescue of the growth of yeast cells transformed with a plasmid in which G22 of TM1 was mutated to charged amino acids, a change that prevents expression of functional CD39, and L489, M492, and G496 of TM2 were randomly mutagenized. The result that the presence of charged or polar amino acids at positions 492 and/or 496 rescued the G22 mutant suggested that this surface of TM2 is close to the surface of TM1 with G22 so that hydrogen bonds or salt linkages could form between the mutated amino acids. If helix mobility is sufficient so that there is no stable contact between helix faces, one would expect that the G22R mutant could be rescued by the presence of Glu at any position within a helical turn of G496. In fact, only the G496E mutation was effective in rescue, while all the other mutants from A491E to F498E were not. This result suggests that there is a stable conformation of the transmembrane helices and that their rotational mobility is a transient phenomenon.

Similarly, the mutation of S24 to Glu was rescued by polar amino acids at A491 even when T495 was changed to Val, indicating that the surface of TM1 with S24 interacts with the surface of TM2 with A491 and T495.

While the experiments of [18] on crosslinking of Cys residues placed in the transmembrane domains suggested considerable helix mobility, they also found that the I33C and A34C mutants in TM1, and the V483C and S480C mutants in TM2 were unable to crosslink under two conditions: low temperature $\left(4^{\circ} \mathrm{C}\right)$ and presence of substrate (ATP), suggesting that the residues are on TM1-TM2 interacting faces and not available for TM1-TM1' and TM2-TM2' interactions and crosslinking.

The results presented here suggest that the face of TM1 including G22 contacts the face of TM2 containing G496 and M492, and that the face of TM1 with S24 contacts the face of TM2 with T495 and A491. Are these interactions between the transmembrane helices intramolecular or intermolecular? We cannot answer this question with these data. It is possible that the two sets of helical interactions represent intramolecular and intermolecular interactions. However, a more likely possibility is that both the G22 TM1-G496 TM2 surfaces and the S24 TM1-T495 TM2 surfaces are involved in intramolecular interactions.

With regard to the latter point, the studies on the structures of the NTPDase proteins by the Strater group [21, 43, 44] have revealed that the molecule undergoes domain closure movement of the extracellular domains on binding of substrate involving a relative rotation of $15^{\circ}$ and up to $21.8^{\circ}$ of the C-terminal domain (II) with respect to the $\mathrm{N}$-terminal domain (I) through an axis between the domains. As [21]

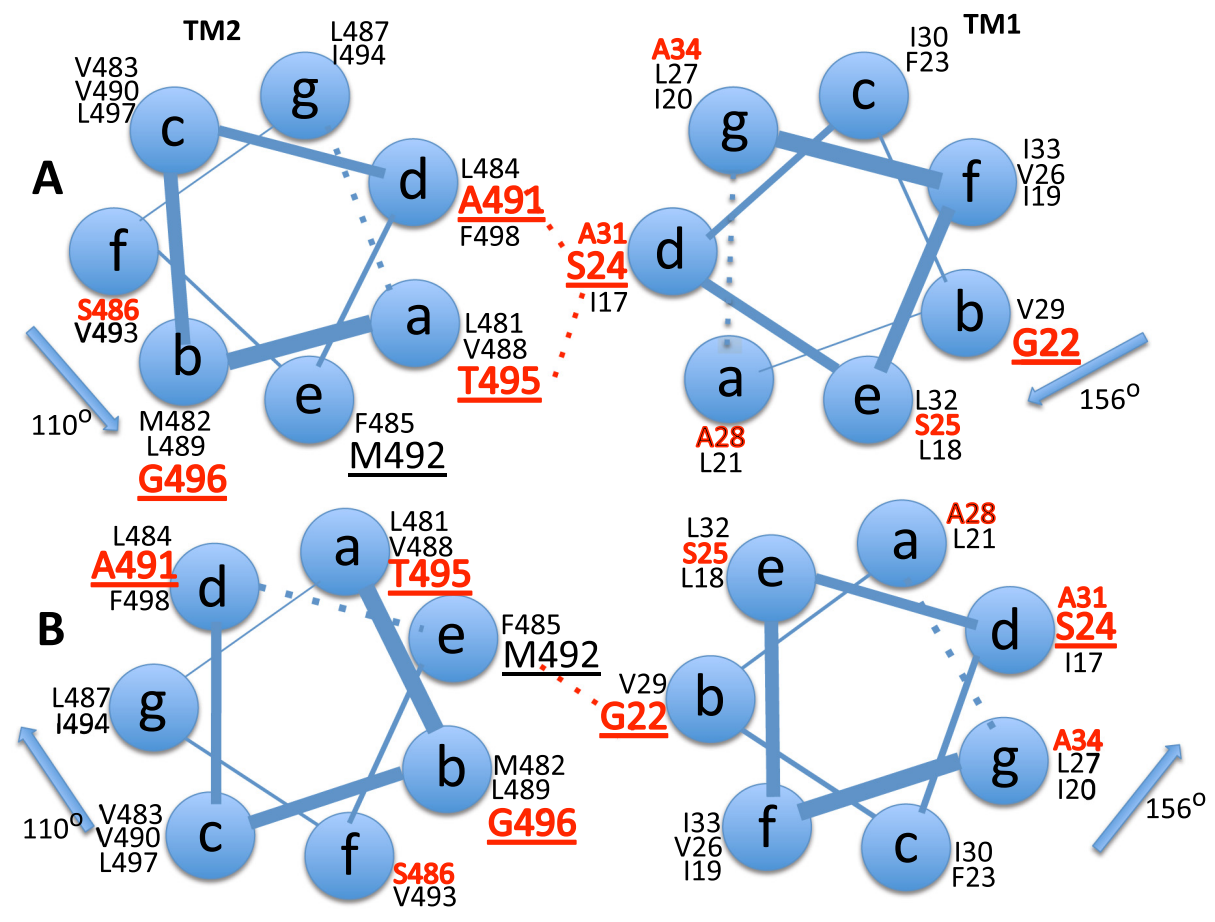

Figure 6. Helical wheel heptad projections (45) of TM1 and TM2 showing the arrangement of the amino acids on the surface of the helix. TM1, starting with A34, and TM2, starting with L481, are shown from the extracellular surface to the cytoplasmic surface of the membrane going into the plane of the page. The residues colored red have helix packing values larger than that of Gln (38). The surfaces of TM1 with G22 and S24 are proposed to interact with the surfaces of TM2 with G496/M492 and T495/A491, respectively. (A) TM domain interactions in the open state and (B) TM domain interactions in the closed state. The arrows indicate the direction of rotation of the TM domains through the stated angles in order for the TM domains to move from one stable interaction to the other. 
have suggested and we have proposed [18] from different perspectives, the dynamic motions of the transmembrane domains observed by us are likely to be coupled to the motions of the two extracellular domains of the NTPDases associated with substrate-binding described by [21, 43]. Accordingly, we suggest that the rotation of the transmembrane domains between the position allowing interaction between the S24TM1 and T495, A491 TM2 surfaces to the position for the interaction between the G22 TM1 and G496, M492-TM2 surfaces, shown by the helical wheel heptad projections [45] in Figure 6, could provide the driving force for or are the consequence of the approximately $20^{\circ}$ rotation of the C-terminal extracellular domain with respect to the N-terminal domain. The observation that CD39 lacking the transmembrane domains has decreased hydrolytic activity [15] suggests that the TM domains are involved in the enzymatic activity and not passively responding to the movement of the extracellular domains. For instance, the rotation of the TM domains between the two stable interacting sites might allow for capture of ATP in the open conformation and catalysis in the closed conformation of the extracellular domains corresponding to the S24 TM1 and T495, A491 TM2 and G22 TM1 and G496, M492 TM2 interactions, respectively.

One might wonder how cumulative rotation of approximately $270^{\circ}$ of the transmembrane domains could result in only a $20^{\circ}$ rotation of the extracellular domains. A likely possibility is the compliance $[46,47]$ of the structures between and including the extracellular domains and the transmembrane domains that can yield elastically to the torque.

In any event, stable interactions between these surfaces of the transmembrane domains could explain the inhibition by ligand and low temperature of intermolecular disulfide crosslinking between the A34C, I33C, V483C, and S480C mutants observed by [18].

Examination of interactions between the transmembrane helices at regions close to the extracellular boundary will provide more information about this question.

\section{ACKNOWLEDGMENTS}

We thank Alison Grinthal and the members of the laboratory for suggestions, comments, and help. This work was supported by grant HL08893 from the National Institutes of Health to GG.

\section{REFERENCES}

[1] Meyer-Fernades JR. Ecto-ATPases in protozoa parasites: looking for function. Parasitol Int. 2002;51(3):299-303. doi:10.1016/ S1383-5769(02)00017-X

[2] Plesner L. Ecto-ATPases: identities and functions. Int Rev Cytol. 1995;158:141-214.

[3] Bigonnesse F, Lévesque SA, Kukulski F, Lecka J, Robson SC, Fernandes MJ, Sévigny J. Cloning and characterization of mouse nucleoside triphosphate diphosphohydrolase-8. Biochemistry. 2004;43(18):5511-9. doi:10.1021/bi0362222
[4] Wang TF, Guidotti G. Golgi localization and functional expression of human uridine diphosphatase. J Biol Chem. 1998;273 (18):11392-9. doi:10.1074/jbc.273.18.11392

[5] Biederbick A, Rose S, Elsässer HP. A human intracellular apyrase-like protein, LALP70, localizes to lysosomal/autophagic vacuoles. J Cell Sci. 1999;112:2473-84.

[6] Shi JD, Kukar T, Wang CY, Li QZ, Cruz PE, Davoodi-Semiromi A, Yang P, Gu Y, Lian W, Wu DH, She JX. Molecular cloning and characterization of a novel mammalian endo-apyrase (LALP1). J Biol Chem. 2001;276(20):17474-8. doi:10.1074/jbc.M0115 69200

[7] Mulero JJ, Yeung G, Nelken ST, Ford JE. CD39-L4 is a secreted human apyrase, specific for the hydrolysis of nucleoside diphosphates. J Biol Chem. 1999;274(29):20064-20067. doi:10.1074/ jbc.274.29.20064

[8] Braun N, Fengler S, Ebeling C, Servos J, Zimmermann H. Sequencing, functional expression and characterization of rat NTPDase6, a nucleoside diphosphatase and novel member of the ecto-nucleoside triphosphate diphosphohydrolase family. Biochem J. 2000;351(3):639-47. doi:10.1042/0264-6021:35 10639

[9] Wang TF, Guidotti G. CD39 is an ecto-(Ca2+,Mg2+)-apyrase. J Biol Chem. 1996;271(17):9898-901. doi:10.1074/jbc.271. 17.9898

[10] Kaczmarek E, Koziak K, Sevigny J, Siegel JB, Anrather J, Beaudoin AR, Bach FH, Robson SC. Identification and characterization of CD39 vascular ATPdiphosphohydrolase. J Biol Chem. 1996;271:33116-22.

[11] Robson SC, Sévigny J, Zimmermann H. The E-NTPDase family of ectonucleotidases: structure function relationships and pathophysiological significance. Purinergic Signal. 2006;2(2): 409-30. doi:10.1007/s11302-006-9003-5

[12] Gayle RB 3rd, Maliszewski CR, Gimpel SD, Schoenborn MA, Caspary RG, Richards C, Brasel K, Price V, Drosopoulos JH, Islam N, Alyonycheva TN, Broekman MJ, Marcus AJ. Inhibition of platelet function by recombinant soluble ecto-ADPase/CD39. J Clin Invest. 1998;101(9):1851-9. doi:10.1172/JCI1753

[13] Maliszewski CR, Delespesse GJ, Schoenborn MA, Armitage RJ, Fanslow WC, Nakajima T, Baker E, Sutherland GR, Poindexter K, Birks C, et al. The CD39 lymphoid cell activation antigen. Molecular cloning and structural characterization. J Immunol. 1994;153:3574-83.

[14] Handa M, Guidotti G. Purification and cloning of a soluble ATPdiphosphohydrolase (apyrase) from potato tubers (Solanum tuberosum). Biochem Biophys Res Commun. 1996;218(3):91623. doi:10.1006/bbrc.1996.0162

[15] Wang TF, Ou Y, Guidotti G. The transmembrane domains of ectoapyrase (CD39) affect its enzymatic activity and quaternary structure. J Biol Chem. 1998;273(38):24814-21doi:10.1074/ jbc.273.38.24814

[16] Grinthal A, Guidotti G. Substitution of His59 converts CD39 apyrase into an ADPase in a quaternary structure dependent manner. Biochemistry. 2000;39:9-16. doi:10.1021/bi991751k

[17] Grinthal A, Guidotti G. Transmembrane domains confer different substrate specificities and adenosine diphosphate hydrolysis mechanisms on CD39, CD39L1, and chimeras. Biochemistry. 2002;41:1964-56. doi:10.1021/bi015563h

[18] Grinthal A, Guidotti G. Dynamic motions of CD39 transmembrane domains regulate and are regulated by the enzymatic active site. Biochemistry. 2004;43(43):13849-58. doi:10.1021/ bi048644x

[19] Grinthal A, Guidotti G. CD39, NTPDase 1, is attached to the plasma membrane by two transmembrane domains. Why? Purinergic Signal. 2006;2(2):391-8. doi:10.1007/s11302-005-5907-8 
[20] Grinthal A, Guidotti G. Bilayer mechanical properties regulate the transmembrane helix mobility and enzymatic state of CD39. Biochemistry. 2007;46(1): 278-90. doi:10.1021/bi061052p

[21] Zebisch M, Krauss M, Schäfer P, Sträter N. Crystallographic evidence for a domain motion in rat nucleoside triphosphate diphosphohydrolase (NTPDase) 1. J Mol Biol. 2012;415(2):288-306. doi:10.1016/j.jmb.2011.10.050

[22] Marinus MG, Carraway M, Frey AZ, Brown L, Arraj JA. Insertion mutations in the dam gene of Escherichia coli K-12. Mol Gen Genet. 1983;192(1-2):288-9. doi:10.1007/BF00327681

[23] Boyum R, Guidotti G. Glucose-dependent, cAMP-mediated ATP efflux from Saccharomyces cerevisiae. Microbiol. 1997;143 (6):1901-8. doi:10.1099/00221287-143-6-1901

[24] Miller JM. A short course in Bacterial Genetics. Cold Spring Harbor Laboratory (NY): Cold Spring Harbor. 439-440.

[25] Sherman F, Fink GR, Hicks JB. Methods in yeast genetics. Gold Spring Harbor Laboratory (NY): Cold Spring Harbor; 1986. 160-175.

[26] Wach A, Brachat A, Pöhlmann R, Philippsen P. New heterologous modules for classical or PCR-based gene disruptions in Saccharomyces cerevisiae. Yeast. 1994;10(13):1793-808. doi:10.1002/yea.320101310

[27] Goldstein AL, McCusker JH. Three new dominant drug resistance cassettes for gene disruption in Saccharomyces cerevisiae. Yeast. 1999;15:1541-53.

[28] Geitz RD, Woods RA. Transformation of yeast by lithium acetate/single-stranded carrier DNA/polyethylene glycol method. Methods Enzymol. 2002;350:87-96. doi:10.1016/S0076-6879 (02)50957-5

[29] van Rijn HJ, Boer P, Steyn-Parvé EP. Biosynthesis of acid phosphatase of baker's yeast. Factors influencing its production by protoplasts and characterization of the secreted enzyme. Biochem Biophys Acta. 1972;268:431-41

[30] Zhong X, Malhotra R, Woodruff R, Guidotti G. Mammalian plasma membrane ecto-nucleoside triphosphate diphosphohydrolase 1, CD39, is not active intracellularly. The N-glycosylation state of CD39 correlates with surface activity and localization. J Biol Chem. 2001;276:41518-25.

[31] Ames BN. Assay of inorganic phosphate, total phosphate and phosphatases. Methods Enzymol. 1966;8:115-8. doi:10.1016/ 0076-6879(66)08014-5

[32] Vernet T, Dignard D, Thomas DY. A family of yeast expression vectors containing the phage f1 intergenic region. Gene. 1987;52(2-3):225-33. doi:10.1016/0378-1119(87)90049-7

[33] Minor DL, Masseling SL, JanYN, Jan LY. Transmembrane structure of an inwardly rectifying potassium channel. Cell. 1999;96 (6):879-91. doi:10.1016/S0092-8674(00)80597-8

[34] Zhong X, Guidotti G. A yeast Golgi E-type ATPase with an unusual membrane topology. J Biol Chem. 1999;274 (46):32704-11. doi:10.1074/jbc.274.46.32704

[35] Maser P, Sutterlin C, Kralli A, Kaminsky R. A nucleoside transporter from Trypanosoma brucei involved in drug resistance. Science. 1999;285(5425):242-4. doi:10.1126/science.285. 5425.242

[36] Knowles AF, Nagy AK. Inhibition of an ecto-ATP-diphosphohydrolase by azide. Eur J Biochem. 1999;262(2):349-57. doi:10.1046/j.1432-1327.1999.00389.x

[37] Eisenberg D, Weiss RM, Terwilliger TC, Wilcox W. Hydrophobic moments and protein structure. Faraday Symp Chem Soc. 1982;17:109-20. doi:10.1039/fs9821700109

[38] Liu W, Eilers M, Patel AB, Smith SO. 2004Helix packing moments reveal diversity and conservation in membrane protein structure. J Mol Biol. 1999;337(3):713-29. doi:10.1016/ j.jmb.2004.02.001

[39] Eilers M, Patel AB, Liu W, Smith SO. Comparison of helix interactions in membrane and soluble alpha-bundle proteins. Biopys J. 2002;82:2720-36.

[40] Dunnill P. The use of helical net-diagrams to represent protein structures. Biophys J. 1968;8(7):865-75. doi:10.1016/S00063495(68)86525-7

[41] Wang TF, Rosenberg PA, Guidotti G. Characterization of brain ecto-apyrase: evidence for only one ecto-apyrase (CD39) gene. Brain Res Mol Brain Res. 1997;47(1-2):295-302. doi:10.1016/ S0169-328X(97)00066-1

[42] Engelman DM, Steitz TA, Goldman A. Identifying nonpolar transbilayer helices in amino acid sequences of membrane proteins. Annu Rev Biophys Biophys Chem. 1986;15(1):321-53. doi:10.1146/annurev.bb.15.060186.001541

[43] Zebisch $M$, Krauss $M$, Schäfer $P$, Lauble $P$, Sträter $N$. Crystallographic snapshots along the reaction pathway of nucleoside triphosphate diphosphohydrolases. Structure. 2013;21 (8):1460-475. doi:10.1016/j.str.2013.05.016

[44] Zimmermann H, Zebisch M, Sträter N. Cellular function and molecular structure of ecto-nucleotidases. Purinergic Signal. 2012;8(3):437-502. doi:10.1007/s11302-012-9309-4

[45] Cohen C, Parry DAD. $\alpha$-Helical coiled coils and bundles: how to design an $\alpha$-helical protein. Proteins. 1990;7(1):1-15. doi:10.1002/prot.340070102

[46] Wächter A, Bi Y, Dunn SD, Cain BD, Sielaff H, Wintermann F, Engelbrecht S, Junge W. Two rotary motors in F-ATP synthase are elastically coupled by a flexible rotor and a stiff stator stalk. Proc Natl Acad Sci U S A. 2011;108:3924-9.

[47] Sielaff H, Rennekamp H, Wachter A, Xie H, Hilbers F, Feldbauer $\mathrm{K}$, Dunn SD, Engelbrecht S, Junge W. Domain compliance and elastic power transmission in rotary $\mathrm{F}(0) \mathrm{F}(1)$-ATPase. Proc Natl Acad Sci U S A. 2008;105(46):17760-5. doi:10.1073/pnas. 0807683105

\section{COMPETING INTERESTS}

The authors declare no competing interests.

\section{PUBLISHING NOTES}

(C) 2014 S. Paavilainen and G. Guidotti. This work has been published open access under Creative Commons Attribution License CC BY 4.0, which permits unrestricted use, distribution, and reproduction in any medium, provided the original work is properly cited. Conditions, terms of use and publishing policy can be found at www.scienceopen.com.

Please note that this article may not have been peer reviewed yet and is under continuous post-publication peer review. For the current reviewing status please click here or scan the QR code on the right.

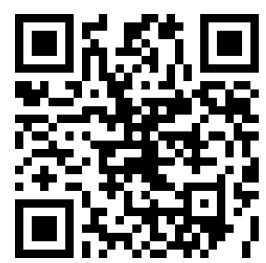

\section{scienceOPEN.com}

research+publishing network 